\title{
(6) OPEN ACCESS \\ Statins are underused in recent-onset Parkinson's disease with increased vascular risk: findings from the UK Tracking Parkinson's and Oxford Parkinson's Disease Centre (OPDC) discovery cohorts
}

\author{
Diane M A Swallow, ${ }^{1}$ Michael A Lawton, ${ }^{2}$ Katherine A Grosset, ${ }^{1}$ Naveed Malek, ${ }^{1}$ \\ Johannes Klein, ${ }^{3}$ Fahd Baig, ${ }^{3}$ Claudio Ruffmann, ${ }^{3}$ Nin P Bajaj, ${ }^{4}$ Roger A Barker, ${ }^{5}$ \\ Yoav Ben-Shlomo, ${ }^{2}$ David J Burn, ${ }^{6}$ Thomas Foltynie, ${ }^{7}$ Huw R Morris, ${ }^{8}$ Nigel Williams, ${ }^{9}$ \\ Nicholas W Wood, ${ }^{10}$ Michele T M Hu, ${ }^{3}$ Donald G Grosset, ${ }^{1}$ on behalf of PRoBaND \\ Clinical Consortium ${ }^{11}$
}

For numbered affiliations see end of article.

\section{Correspondence to} Dr Diane Swallow, Institute of Neurological Sciences, Queen Elizabeth University Hospital, Glasgow G51 4TF, UK; diane. swallow@nhs.net

Received 29 March 2016 Revised 22 June 2016 Accepted 17 July 2016

\section{CrossMark}

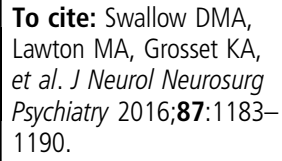

\section{ABSTRACT}

Background Cardiovascular disease (CVD) influences phenotypic variation in Parkinson's disease (PD), and is usually an indication for statin therapy. It is less clear whether cardiovascular risk factors influence PD phenotype, and if statins are prescribed appropriately. Objectives To quantify vascular risk and statin use in recent-onset $\mathrm{PD}$, and examine the relationship between vascular risk, PD severity and phenotype.

Methods Cardiovascular risk was quantified using the QRISK2 calculator (high $\geq 20 \%$, medium $\geq 10$ and $<20 \%$, low risk $<10 \%$ ). Motor severity and phenotype were assessed using the Movement Disorder Society Unified PD Rating Scale (UPDRS) and cognition by the Montreal cognitive assessment.

Results In 2909 individuals with recent-onset PD, the mean age was 67.5 years (SD 9.3), 63.5\% were men and the mean disease duration was 1.3 years (SD 0.9). $33.8 \%$ of cases had high vascular risk, $28.7 \%$ medium risk, and $22.3 \%$ low risk, while $15.2 \%$ of cases had established CVD. Increasing vascular risk and CVD were associated with older age $(p<0.001)$, worse motor score $(p<0.001)$, more cognitive impairment $(p<0.001)$ and worse motor phenotype $(p=0.021)$. Statins were prescribed in $37.2 \%$ with high vascular risk, $15.1 \%$ with medium vascular risk and $6.5 \%$ with low vascular risk, which compared with statin usage in $75.3 \%$ of those with CVD.

Conclusions Over $60 \%$ of recent-onset PD patients have high or medium cardiovascular risk (meriting statin usage), which is associated with a worse motor and cognitive phenotype. Statins are underused in these patients, compared with those with vascular disease, which is a missed opportunity for preventive treatment. Trial registration number GN11NE062, NCT02881099.

\section{BACKGROUND}

Parkinson's disease (PD) and cardiovascular disease (CVD) become more prevalent with advancing age. CVD is therefore likely to affect a large number of individuals with PD. A variety of clinical, imaging and pathological studies in elderly individuals without PD, ${ }^{12}$ as well as smaller PD studies, ${ }^{3-10}$ show links between established vascular disease and vascular risk factors, and gait and cognitive impairment. A combination of Lewy body and vascular pathology may create a mixed clinical phenotype, and explain some of the variation in the responsiveness of the motor and cognitive features to antiparkinsonian therapy.

Vascular preventive treatment is well established. Primary prevention is recommended when an individual's calculated 10 -year vascular risk is $10 \%$ or more ${ }^{11}$ and involves the use of cholesterol-lowering therapy (mainly with HMG-CoA reductase inhibitors, commonly referred to as statins) and management of other vascular risk factors such as hypertension. Secondary prevention (after a vascular event) similarly involves the use of statins, as well as antihypertensive and antiplatelet therapy. Additionally, statins are of particular interest in PD as possible neuroprotectants, given their beneficial role in the attenuation of inflammatory responses, including the production of tumour necrosis factor $\alpha$, nitric oxide and superoxide; the reduction in the accumulation of $\alpha$-synuclein; and alteration of dopamine D1/D2 receptor modulation. ${ }^{12}$ However, varying rates of statin usage are reported in patients with vascular risk and vascular disease, ${ }^{13-18}$ and it is not known whether PD patients have equitable access to statins.

We therefore studied cardiovascular risk and CVD rates in recent-onset $\mathrm{PD}$, in relation to clinical phenotype, more specifically the motor and cognitive features, and the use of statins in these patients.

\section{METHODS}

\section{Participants}

Study participants were enrolled prospectively in either the UK Tracking Parkinson's study, or the Oxford Discovery study. Participants were recruited from February 2012 to May 2014 in the Tracking Parkinson's study and from September 2010 to October 2015 in the Oxford Discovery study. Our analysis is based on the baseline data from these large multicentre studies, whose protocols including inclusion/exclusion criteria are detailed elsewhere. ${ }^{19} 20$ In brief, in both studies, cases with a clinical diagnosis of PD were recruited, fulfilling 
Queen Square Brain Bank criteria, with written consent, approval of multicentre regional ethics committees and in compliance with national legislation and the Declaration of Helsinki. For the current analysis, cases with normal functional dopaminergic imaging performed after study entry, and cases with a revised diagnosis at their latest follow-up visit, were excluded. When assessing the clinical correlates of CVD, we further excluded cases with any features that were possibly atypical or unusual at baseline assessment, including an unusual presentation, symptom, sign, progression or response to medication, to reduce any effect from a possible alternative diagnosis than PD (eg, vascular parkinsonism).

\section{Measurement instruments}

Established vascular diagnoses and risk factors were collected from self-report completed at clinic attendance, and was performed at the same time as, and therefore with input from, physician/nurse access to medical records. The individual vascular factors collected are the standard risk factor variables recommended by the National Institute of Clinical Excellence (NICE) in the UK to clinically assess vascular risk. ${ }^{21}$ The 10 -year future cardiovascular risk was then calculated using the QRISK2-2015 prediction algorithm, ${ }^{22}$ also recommended by NICE, ${ }^{11}$ which computes risk based on demographic and comorbid vascular features for example, age, smoking status, ethnicity, systolic blood pressure, body mass index, treated hypertension and type 2 diabetes. The calculation is only appropriate in patients aged under 85 years and without a previous vascular event. Since national treatment thresholds were reduced recently from $20 \%$ to $10 \%$ calculated risk, ${ }^{11}$ statin implementation was examined against both of these thresholds, and designated as high $(\geq 20 \%)$, medium $(\geq 10$ and $<20 \%)$ or low $(<10 \%)$ risk. Vascular preventive medications, including lipid-lowering, antiplatelet, anticoagulant and antihypertensive therapies, were identified from medication histories, using British National Formulary classifications.

Motor function was scored according to Part 3 of the Movement Disorder Society Unified PD Rating Scale (UPDRS 3), and was used to define motor subtype using a predetermined formula which uses these variables to define tremor dominant (TD) and postural instability gait difficulty (PIGD) phenotypes. ${ }^{23}$ Cognition was assessed by the Montreal cognitive assessment (MoCA), adjusted for education years according to standard methods ( 1 point added to the total score if education years were $\leq 12$, to a maximum score of 30 ) and categorised as normal cognition (24-30), mild cognitive impairment (22-23) or dementia $(<22) .{ }^{24}$ Levodopa equivalent daily dose (LEDD) was calculated using an established formula. ${ }^{25}$

\section{Statistical analysis}

Generalised linear modelling was used to assess clinical variables across vascular risk categories, adjusting for multiple covariates (age, sex, disease duration and coffee use), with heterogeneity and trend $\mathrm{p}$ values calculated when there were more than two categories. The linearity of the continuous confounders (age and disease duration) was tested using fractional polynomials in univariate models and then transformed if they showed evidence of non-linearity. Regression models used were: multinomial logistic for motor subtype analysis (using TD as the baseline); ordered logistic for categorised MoCA and smoking status; linear for age, disease duration, MoCA total, LEDD and UPDRS 3; and logistic for sex. The main analysis excluded those with a revised diagnosis, those diagnosed $>3.5$ years ago and those without available QRISK2 prediction algorithm or medication data. An additional sensitivity analysis was performed imputing missing outcome and exposure data. In this analysis, MoCA, motor phenotype and UPDRS 3 scores were calculated using expected scores where at least $80 \%$ of the questions in each scale were answered. Any remaining missing data were imputed using the chained equation approach to multiple imputation, creating 10 imputed data sets. Adjusted $\mathrm{p}$ values of $<0.05$ were considered significant. IBM SPSS Statistics for Windows, V.22.0, Armonk, New York, USA, and STATA V.13 were used.

\section{RESULTS}

Out of 3019 cases (Tracking Parkinson's 2006, Oxford Discovery 1013), 110 (3.6\%) were excluded for the following reasons: revised diagnosis $(n=32)$, disease duration $>3.5$ years $(n=49)$, missing statin usage $(n=5)$ and missing QRISK2 status $(n=24$; figure 1$)$. In the remaining 2909 cases, the mean age was 67.5 years (SD 9.3), mean disease duration 1.3 years (SD 0.9 ) and $65.3 \%$ were men. Further baseline characteristics are summarised in table 1.

Established vascular disease was present in $15.2 \%$ of cases (table 2). Hypertension was present in $34.4 \%$, high cholesterol in $32.4 \%$, diabetes in $8.6 \%$ and current cigarette smoking in $3.2 \%$. Antihypertensive medication was the most commonly prescribed preventive cardiovascular therapy at $39.5 \%$, while lipid-lowering medication was prescribed in 30.4\%. Statins were the most common lipid-lowering medication $(97.9 \%$ of cases on lipid-lowering therapy). Other cardiovascular risk factors and preventive medications are listed in table 2.

The QRISK2 score (ie, 10-year future cardiovascular risk) was low in 648 cases $(22.3 \%)$, medium in 836 cases $(28.7 \%)$ and high in 984 cases $(33.8 \%$; table 3$)$. Statins were prescribed in a minority of those with a vascular risk indication for their use (15.1\% of those with medium vascular risk, and $37.2 \%$ of cases with high vascular risk). In contrast, statins were prescribed in $75.3 \%$ of cases with established vascular disease.

The relationship between graded vascular risk and vascular disease, and the clinical characteristics of PD was analysed in the 2611 cases without any features that might possibly indicate

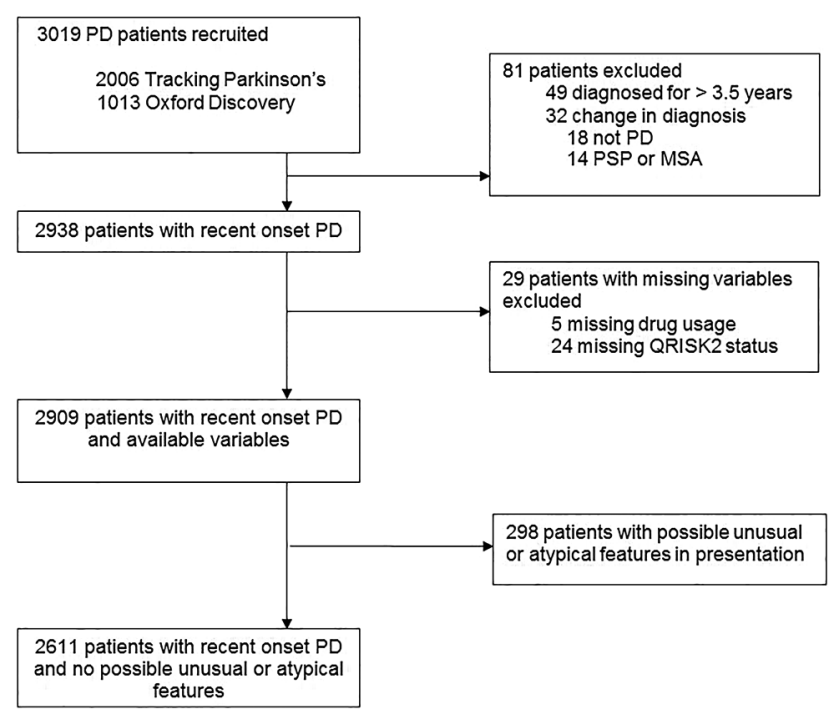

Figure 1 Disposition of cases recruited to the study, and reasons for exclusion. The main analysis was performed in 2909 cases; clinical correlates were examined in 2611 cases, to reduce any effect of possible diagnostic inaccuracy. PD, Parkinson's disease, PSP, progressive supra nuclear palsy, MSA, multiple system atrophy. 
Table 1 Demographic and clinical characteristics of 2909 recent-onset Parkinson's disease cases

\begin{tabular}{lc}
\hline Variable & Result \\
\hline Age & $67.5(9.3)$ \\
Male sex & $1898(65.3 \%)$ \\
Disease duration (years) & $1.3(0.9)$ \\
UPDRS 3 & $24.2(12.1)$ \\
Motor subtype & \\
TD & $1303(48.1 \%)$ \\
PIGD & $1061(39.2 \%)$ \\
Indeterminate & $344(12.7 \%)$ \\
MoCA & \\
Total score & $25.1(3.5)$ \\
Normal & $1972(72.1 \%)$ \\
MCI & $370(13.5 \%)$ \\
Dementia & $394(14.4 \%)$ \\
Antiparkinsonian medication & \\
Drug naïve & $314(10.8 \%)$ \\
Levodopa & $1776(61.2 \%)$ \\
Dopamine agonist & $873(30.0 \%)$ \\
MAOB inhibitor & $723(24.9 \%)$ \\
COMT inhibitor & $67(2.3 \%)$ \\
Anticholinergic & $38(1.3 \%)$ \\
Amantadine & $28(1.0 \%)$ \\
LEDD & \\
\hline Data are mean (SD) or number (percentage). & \\
COMT, catechol-O-methyl transferase; LEDD, levodopa equivalent daily & dose; MAOB, \\
monoamine oxidase type B; MCI, mild cognitive impairment; MoCA, Montreal \\
Cognitive assessment; PIGD, postural instability gait difficulty; TD, tremor dominant; \\
UPDRS 3, Movement Disorder Society unified Parkinson's disease rating scale part 3. \\
\end{tabular}

an alternative diagnosis than PD (table 4). This meant that 298 cases were excluded from this analysis $(10.2 \%$ of the 2909 study group). The analysis also excluded cases with missing results for disease duration $(n=6,0.2 \%)$, UPDRS $3(n=189$, $7.2 \%)$, motor subtype $(n=185,7.1 \%)$, MoCA $(n=157,6.0 \%)$ and LEDD $(n=20,0.8 \%)$. Additional analysis using multiple imputation of this missing data showed no qualitative differences, aside from the LEDD comparison in statin versus nonstatin users (results not shown).

Increasing vascular risk was associated with increasing age $(\mathrm{p}<0.001)$ and an increasing proportion of men $(\mathrm{p}<0.001)$. Increasing vascular risk was also associated with worsening UPDRS 3 scores when adjusted for age, sex, disease duration and coffee intake $(\mathrm{p}<0.001)$, with UPDRS 3 scores ranging from 20.3 (SD 10.7) in those with a low QRISK2 score to 26.8 (SD 12.3) in those with established vascular disease. Increasing vascular risk was similarly associated with an increasing proportion with the PIGD phenotype $(\mathrm{p}=0.021)$ rising from $32.3 \%$ in those with a low QRISK2 score to $48.0 \%$ of cases with established vascular disease. Increasing vascular risk was also associated with increasing cognitive impairment; a worsening MoCA score $(p<0.001)$ as well as an increasing proportion with MCI and dementia $(p=0.008)$. The motor and cognitive characteristics of cases with high vascular risk were very similar to those seen in cases with established vascular disease (table 4). When we considered the potential effects of the interaction of sex on the clinical correlates of each vascular risk category, we did not find any statistically significant interactions (data not shown).

When we compared the clinical features of individuals treated versus untreated with statins (all indications), statin users had
Table 2 Vascular disease, risk factors and treatment in 2909 recent-onset Parkinson's disease cases

\begin{tabular}{|c|c|}
\hline Variable & Result \\
\hline Vascular diagnosis & $441(15.2 \%)$ \\
\hline Angina & $269(9.3 \%)$ \\
\hline Myocardial infarction & $134(4.6 \%)$ \\
\hline Transient ischaemic attack/stroke & $150(5.2 \%)$ \\
\hline \multicolumn{2}{|l|}{ Vascular risk factors } \\
\hline Diabetes & $249(8.6 \%)$ \\
\hline Type 1 & $13(0.5 \%)$ \\
\hline Type 2 & $236(8.2 \%)$ \\
\hline High cholesterol & $936(32.4 \%)$ \\
\hline Hypertension & $992(34.4 \%)$ \\
\hline Rheumatoid arthritis & $69(2.4 \%)$ \\
\hline Body mass index $\left(\mathrm{kg} / \mathrm{m}^{2}\right)$ & $27.1(4.7)$ \\
\hline Blood pressure, systolic/diastolic & $140(20) / 80(11)$ \\
\hline Orthostatic hypotension & $525(18.5 \%)$ \\
\hline \multicolumn{2}{|l|}{ Smoking } \\
\hline Non-smoker & $1539(57.9 \%)$ \\
\hline Ex-smoker & $1023(38.5 \%)$ \\
\hline Current smoker & $96(3.2 \%)$ \\
\hline Light & $48(1.8 \%)$ \\
\hline Moderate & $33(1.3 \%)$ \\
\hline Heavy & $15(0.6 \%)$ \\
\hline \multicolumn{2}{|l|}{ Current coffee intake (cups per day) } \\
\hline Less than 1 & $257(9.6 \%)$ \\
\hline 1 & $194(7.2 \%)$ \\
\hline $2-3$ & $719(26.7 \%)$ \\
\hline 4 or more & $1522(56.5 \%)$ \\
\hline \multicolumn{2}{|l|}{ Cardiovascular medication } \\
\hline Lipid lowering & $885(30.4 \%)$ \\
\hline Statins & $866(97.9 \%)^{*}$ \\
\hline Other & $19(2.1 \%)^{*}$ \\
\hline Antihypertensive & $1150(39.5 \%)$ \\
\hline Antiplatelet & $430(14.8 \%)$ \\
\hline Anticoagulant & $122(4.2 \%)$ \\
\hline
\end{tabular}

*Percentage of total lipid lowering.

Data are mean (SD) or number (percentage).

Table 3 Vascular preventive medication usage according to indication in 2909 recent-onset Parkinson's disease cases

\begin{tabular}{|c|c|c|c|c|}
\hline \multirow[b]{2}{*}{ Medication } & \multicolumn{3}{|c|}{ Primary prevention } & \multirow{2}{*}{$\begin{array}{l}\text { Secondary } \\
\text { prevention }\end{array}$} \\
\hline & $\begin{array}{l}\text { QRISK2 } \\
<10 \% \\
=648 \\
(22.3 \%)\end{array}$ & $\begin{array}{l}\text { QRISK2 } \\
\geq 10 \% \text { and } \\
<20 \% \\
n=836 \\
(28.7 \%)\end{array}$ & $\begin{array}{l}\text { QRISK2 } \\
\geq 20 \% \\
\mathrm{n}=984 \\
(33.8 \%)\end{array}$ & \\
\hline Statin & $42(6.5 \%)$ & $126(15.1 \%)$ & 366 (37.2\%) & $332(75.3 \%)$ \\
\hline Antihypertensive & 96 (14.8\%) & $236(28.2 \%)$ & 533 (54.2\%) & 345 (78.2\%) \\
\hline $\begin{array}{l}\text { Antiplatelet/ } \\
\text { anticoagulant }\end{array}$ & $15(2.3 \%)$ & $69(8.3 \%)$ & $180(18.3 \%)$ & 279 (63.3\%) \\
\hline
\end{tabular}

Data are number (percentage). QRISK2 is the 10-year future calculated cardiovascular risk.

less PIGD $(p=0.002)$ but a lower total MoCA score $(p<0.001)$ and a greater proportion with cognitive impairment $(p=0.010)$. Statin users also had a greater LEDD $(p=0.035)$, but, as described earlier, this was not significant in our multiple imputation analysis (table 5). 
Table 4 Clinical correlates in 2611 recent-onset Parkinson's disease cases according to the presence of future cardiovascular risk and existing cardiovascular disease

\begin{tabular}{|c|c|c|c|c|c|c|}
\hline Variable & $\begin{array}{l}\text { QRISK2 } \\
<10 \% \\
n=590(22.6 \%)\end{array}$ & $\begin{array}{l}\text { QRISK2 } \\
\geq 10 \% \text { and }<20 \% \\
n=760(29.1 \%)\end{array}$ & $\begin{array}{l}\text { QRISK2 } \\
\geq 20 \% \\
n=886(33.9 \%)\end{array}$ & $\begin{array}{l}\text { Vascular disease } \\
n=375(14.4 \%)\end{array}$ & Unadjusted $p$ value & Adjusted $p$ value \\
\hline Age & $55.9(7.0)$ & $65.9(4.5)$ & $74.2(5.3)$ & $72.4(7.6)$ & $<0.001$ & $<0.001^{*}$ \\
\hline Male sex & $233(39.5 \%)$ & $482(63.4 \%)$ & $698(78.8 \%)$ & $284(75.7 \%)$ & $<0.001$ & $<0.001 \dagger$ \\
\hline Disease duration & $1.3(0.9)$ & $1.3(0.9)$ & $1.3(0.9)$ & $1.4(0.9)$ & 0.05 & $0.78 \ddagger$ \\
\hline UPDRS 3 & $20.3(10.7)$ & $22.2(10.9)$ & $25.9(12.1)$ & $26.8(12.3)$ & $<0.001$ & $<0.001 \S$ \\
\hline \multicolumn{7}{|l|}{ Motor subtype } \\
\hline TD & $299(54.6 \%)$ & $363(51.0 \%)$ & $397(48.2 \%)$ & $137(40.1 \%)$ & & \\
\hline PIGD & $177(32.3 \%)$ & $248(34.8 \%)$ & $330(40.0 \%)$ & $164(48.0 \%)$ & $<0.001$ & $0.021 \S$ \\
\hline Indeterminate & $72(13.1 \%)$ & $101(14.2 \%)$ & $97(11.8 \%)$ & $41(12.0 \%)$ & 0.60 & $0.069 \S$ \\
\hline \multicolumn{7}{|l|}{$\mathrm{MoCA}$} \\
\hline Total score & $26.6(2.7)$ & $25.6(3.2)$ & $24.2(3.6)$ & $24.1(3.5)$ & $<0.001$ & $<0.001 \S$ \\
\hline Normal & $486(88.0 \%)$ & $569(79.7 \%)$ & $511(61.6 \%)$ & $216(60.3 \%)$ & $<0.001$ & $0.008 \S$ \\
\hline $\mathrm{MCl}$ & $38(6.9 \%)$ & $77(10.8 \%)$ & $147(17.7 \%)$ & $64(17.9 \%)$ & & \\
\hline Dementia & $28(5.1 \%)$ & $68(9.5 \%)$ & $172(20.7 \%)$ & $78(21.8 \%)$ & & \\
\hline LEDD & $273(215)$ & $287(222)$ & $291(190)$ & $314(202)$ & 0.004 & $0.069 \S$ \\
\hline Smoking & & & & & $<0.001$ & $<0.001 \S$ \\
\hline Non-smoker & $413(76.3 \%)$ & $415(58.9 \%)$ & $411(50.4 \%)$ & $158(47.7 \%)$ & & \\
\hline Ex-smoker & $108(20.0 \%)$ & $265(37.6 \%)$ & $375(46.0 \%)$ & $160(48.3 \%)$ & & \\
\hline Current smoker & $20(3.7 \%)$ & $25(3.5 \%)$ & $30(3.7 \%)$ & $13(3.9 \%)$ & & \\
\hline
\end{tabular}

Data are mean (SD) or number (percentage) unless otherwise stated.

${ }^{*}$ Adjusted for disease duration and sex.

†Adjusted for age and disease duration.

¥Adjusted for age and sex.

$\S$ Adjusted for age, sex, disease duration and coffee intake.

LEDD, levodopa equivalent daily dose; MCI, mild cognitive impairment; MoCA, Montreal cognitive assessment; PIGD, postural instability gait difficulty; TD, tremor dominant; UPDRS 3,

Movement Disorder Society unified Parkinson's disease rating scale part 3.

When we compared the clinical features of those using and not using statins in individuals with established vascular disease and a QRISK2 score $\geq 10 \%$ (table 6), those treated with statins in the QRISK2 $\geq 10 \%$ subgroup had less PIGD ( $\mathrm{p}=0.009$ ), but also lower total MoCA scores and a greater proportion with cognitive impairment $(p<0.001)$. Conversely in those with established CVD, those treated with statins were better cognitively, but with no significant differences in the proportion with PIGD.

\section{DISCUSSION}

We have found, in a large study of recent-onset PD, that over $60 \%$ of cases have increased cardiovascular risk (without a history of vascular disease) that places them in a recommended treatment category for statin therapy. However, only around a quarter of those cases $(27.0 \%)$ were prescribed such therapy. In contrast, fewer patients (around 15\%) have manifest CVD, but a much larger proportion of these cases $(75.3 \%)$ are prescribed statins. Given that statins are indicated in both groups, ${ }^{11}$ the comparatively lower usage of statins in those with increased vascular risk, compared with those with manifest CVD, suggests that the assessment and/or treatment approach to vascular risk is fundamentally different from that of manifest vascular disease, in recent-onset PD patients. The clinical relevance of this is suggested by the significant association of vascular risk, in addition to established CVD, with greater motor severity, including more axial features and gait problems, and with cognitive problems, including mild cognitive impairment and dementia.

The effects we have observed relating vascular risk to clinical phenotype in PD extend the evidence linking vascular risk factors with worse neurological status in prior smaller studies.
PD cases with diabetes had worse global cognition, ${ }^{5}$ greater axial impairment ${ }^{6}$ and more rapid progression in terms of motor scores, ${ }^{7}$ while PD cases with hypertension had worse executive function and delayed memory. ${ }^{4}$ Further, carotid artery intima-medial thickness (a marker of subclinical vascular disease) correlated with higher levels of motor and cognitive impairment; ${ }^{26}$ and the presence of one or more vascular risk factor was associated with greater cognitive impairment and motor severity. ${ }^{27}$ Similar observations relate vascular risk to worse neurological status in Alzheimer's disease $(\mathrm{AD})^{28}$ and multiple sclerosis, ${ }^{29}$ so these effects in PD are not unique.

Our use of a combined cardiovascular risk assessment tool is the subject of only one other PD study ${ }^{30}{ }^{31}$ to the best of our knowledge, which used a simplified version of the Framingham risk score. In that study, in 61 cases with an elevated Framingham risk, the Timed Up and Go test was significantly slower than in 22 cases with normal scores, ${ }^{30}$ and increased Framingham scores correlated with the axial motor impairment, ${ }^{31}$ again in keeping with the relationship between vascular risk and motor pattern in our study. Unlike the current study however, they found no relationship between vascular risk and motor or cognitive scoring, perhaps because of their longer PD duration (5.5 years) and considerably smaller study size. ${ }^{30}$ Considering dopaminergic therapy, one recent study found that PD cases with preceding diabetes were prescribed larger doses than cases without preceding diabetes. ${ }^{32}$ We did not find any association between LEDD and vascular risk, even after adjustment for patient age (which may influence drug dosage), despite their greater motor severity. We plan to test whether this is associated with lesser dopaminergic responsiveness in patients with vascular risk, in the ongoing follow-up phase of our study, which includes formal measurement of the L-dopa response. 
Table 5 Clinical correlates in 2611 recent-onset Parkinson's disease cases according to the use of statin medication

\begin{tabular}{lccll}
\hline Variable & $\begin{array}{l}\text { Statin use, } \\
\mathbf{n}=769\end{array}$ & $\begin{array}{l}\text { No statin } \\
\text { use, } \mathbf{n}=1842\end{array}$ & $\begin{array}{l}\text { Unadjusted } \\
\mathbf{p} \text { value }\end{array}$ & $\begin{array}{l}\text { Adjusted } \\
\mathbf{p} \text { value }\end{array}$ \\
\hline Age & $71.1(7.4)$ & $65.8(9.4)$ & $<0.001$ & $0.16^{*}$ \\
Sex & $573(74.5 \%)$ & $1124(61.0 \%)$ & $<0.001$ & $0.52 \dagger$ \\
Disease duration & $1.3(0.9)$ & $1.3(0.9)$ & 0.34 & $0.96 \ddagger$ \\
UPDRS 3 & $25.3(11.9)$ & $23.0(11.6)$ & $<0.001$ & $0.97 \S$ \\
Motor subtype & & & & \\
$\quad$ TD & $361(50.1 \%)$ & $835(49.0 \%)$ & & \\
PIGD & $268(37.2 \%)$ & $651(38.2 \%)$ & 0.61 & $0.002 \S$ \\
$\quad$ Mixed & $92(12.8 \%)$ & $219(12.8 \%)$ & 0.84 & $0.75 \S$ \\
MoCA & & & & \\
$\quad$ Total & $24.2(3.7)$ & $25.5(3.3)$ & $<0.001$ & $<0.001 \S$ \\
$\quad$ Normal & $450(62.2 \%)$ & $1332(76.9 \%)$ & $<0.001$ & $0.010 \S$ \\
$\quad$ MCI & $120(16.6 \%)$ & $206(11.9 \%)$ & & \\
$\quad$ Dementia & $153(21.2 \%)$ & $193(11.1 \%)$ & & \\
$\quad$ LEDD & $291(209)$ & $288(207)$ & 0.71 & $0.035 \S$ \\
Smoking & & & & \\
$\quad$ Non-smoker & $352(50.7 \%)$ & $1045(61.5 \%)$ & $<0.001$ & $0.82 \S$ \\
$\quad$ Ex-smoker & $319(46.0 \%)$ & $589(34.7 \%)$ & & \\
Current smoker & $23(3.3 \%)$ & $65(3.8 \%)$ & & \\
\hline
\end{tabular}

Data are mean (SD) or number (percentage) unless otherwise stated.

${ }^{*}$ Adjusted for disease duration, sex and presence of existing cardiovascular disease or future cardiovascular risk.

tAdjusted for age, disease duration and presence of existing cardiovascular disease or future cardiovascular risk.

$\ddagger$ Adjusted for age, sex and presence of existing cardiovascular disease or future cardiovascular risk.

$\S$ Adjusted for age, sex, disease duration, coffee use and presence of existing cardiovascular disease or future cardiovascular risk.

$\mathrm{MCl}$, mild cognitive impairment; MoCA, Montreal cognitive assessment; PIGD, postural instability gait difficulty; TD, tremor dominant; UPDRS3, Movement Disorder Society unified Parkinson's disease rating scale part 3.

The usage of statins in PD can be compared with that in other patient groups, although there are differences in methodology between the studies. In UK primary care, $80 \%$ of high vascular risk cases (Framingham score $>20 \%$ ) were treated with statins, which is more than double the $37.2 \%$ rate in our PD cases with similar risk (QRISK2 $\geq 20 \%$ ). However, in that study, only $43 \%$ of individuals had a calculable vascular risk, and the $80 \%$ proportion on statins refers to this subset of cases and is therefore artificially high. ${ }^{13}$ In a Swiss study, lipid-lowering therapy was prescribed in $71 \%$ of high-risk cases, although this included a mix of cases with established CVD and high vascular risk scores. ${ }^{18}$ That study also described a medium-risk group (10-20\% vascular risk), of whom 48\% were prescribed statins, much higher than our rate of $15.1 \%$ for a comparable risk level. Although based on disease markers rather than calculated risk, higher statin usage was also reported in a European crosssectional study: $42.2 \%$ with diabetes and $47.0 \%$ with high cholesterol. $^{16}$

The rate of statin use in our PD cases with established CVD $(75.3 \%)$ is very similar to the $74 \%$ rate of statin use for comparable cases in UK primary care, ${ }^{13}$ and higher than that observed in other settings: $68.6 \%$ of Irish community-living adults, ${ }^{17}$ and $43.4 \%$ of Italian patients after myocardial infarction. ${ }^{15}$

Overall, there appear to be higher rates of statin use in cases with manifest CVD than cases with increased vascular risk, which matches our findings in PD cases. Some more specific PD factors may influence this pattern. First, it is possible that the low cigarette smoking rate in PD cases may mislead the patient or clinician when considering vascular risk. Second, muscle cramps are a recognised barrier to statin use and occur in 5\% of the general population, ${ }^{33}$ but may be contributed by PD symptoms; there may be a lower tolerance to statins in patients with such symptoms and increased vascular risk, compared with those with manifest CVD. Preliminary findings from the Parkinson's Pain Study suggest that around 13\% of early PD patients experience painful cramps (Dr Monty Silverdale, personal communication) which may affect statin use. Although the complexity of many antiparkinsonian drug regimens, and other comorbidities, can lead to a high pill burden, which is known to affect medication usage, this does not readily explain the differences in statin use comparing our vascular risk and CVD cases. It may be that, in the absence of manifest CVD, clinicians primarily focus on $\mathrm{PD}$ rather than considering opportunistic vascular preventive treatments. Further analysis of reasons for the nonimplementation or early cessation of statins in PD was beyond the scope of the current project, but merits specific study.

The potential implications of undertreating vascular risk in PD require consideration. If vascular risk is undertreated in PD as a result of statin underutilisation, this could influence the frequency of vascular disease seen in a PD population, which may explain some of the variability in studies comparing the frequency of CVD and risk factors in PD and controls. This could in turn influence conclusions relating to the aetiological role of vascular disease in PD. Vascular preventive therapy primarily reduces acute vascular events, for example, stroke and myocardial infarction, ${ }^{11}$ but may also limit chronic vascular damage. Following stroke, statins reduced white matter hyperintensity progression rates, and limited the decline in executive function. ${ }^{34}$ In $\mathrm{AD}$, observational data suggested that treating vascular risk factors altered the rate of cognitive decline, although one can argue such studies are subject to bias. ${ }^{35}$ Improving the implementation of such treatments may therefore limit a vascular component of motor and cognitive deterioration in PD. However, there are limited data on such effects in PD. In one study, the presence of hypertension in PD patients correlated with greater Hoehn and Yahr stage progression over 5 years. ${ }^{27}$ In the ongoing follow-up phase of our study, we will test whether vascular risk and vascular disease contribute to the evolution of phenotype from TD to PIGD found in earlier longitudinal PD studies. ${ }^{36}$

The observation that individuals with the same vascular risk, but who were treated with statins, had a smaller proportion with the PIGD phenotype than those untreated with statins may lend support to the potential benefits of treating established CVD and elevated cardiovascular risk in PD. However, in those with a QRISK2 score $\geq 10 \%$, those treated with statins had more cognitive impairment compared with those who were untreated. One possible explanation for this is a positive selection bias for starting statins, or maintaining them, when cognition is more impaired, or risks are perceived to be greater (such as in men). Although there are potential mechanisms whereby statins might increase cognitive impairment (through interference with myelin formation and function, ${ }^{37}$ and reduction in coenzyme- $Q_{10}$ levels leading to impaired mitochondrial functioning and increased oxidative stress ${ }^{38}$ ), a recent systematic review and meta-analysis did not find any evidence of significant adverse effects of statins on cognition, either in cognitively normal participants or in those with AD. ${ }^{39}$ In addition, a reverse effect was seen in our study in those with established vascular disease, where those not using statins were cognitively worse. Given that our study is observational, we cannot reach definite conclusions and therefore the results of the Simvastatin as a Neuroprotective Treatment for Moderate Parkinson's Disease (PD STAT) randomised placebo-controlled trial will be 
Table 6 Clinical correlates in 2611 recent-onset Parkinson's disease cases according to the use of statin medication stratified by the presence of future cardiovascular risk and existing cardiovascular disease

\begin{tabular}{|c|c|c|c|c|c|c|c|c|}
\hline \multirow[b]{2}{*}{ Variable } & \multicolumn{4}{|c|}{ Vascular disease, $n=375$} & \multicolumn{4}{|c|}{ QRISK2 $\geq 10 \%, n=1646$} \\
\hline & $\begin{array}{l}\text { Statin use, } \\
n=280\end{array}$ & $\begin{array}{l}\text { No statin } \\
\text { use, } n=95\end{array}$ & $\begin{array}{l}\text { Unadjusted } \\
\text { p value }\end{array}$ & $\begin{array}{l}\text { Adjusted } \\
\mathrm{p} \text { value }\end{array}$ & $\begin{array}{l}\text { Statin use, } \\
n=450\end{array}$ & $\begin{array}{l}\text { No statin } \\
\text { use, } n=1196\end{array}$ & $\begin{array}{l}\text { Unadjusted } \\
\mathrm{p} \text { value }\end{array}$ & $\begin{array}{l}\text { Adjusted } \\
\mathrm{p} \text { value }\end{array}$ \\
\hline Age & $72.5(7.2)$ & $72.0(8.7)$ & 0.57 & $0.60^{*}$ & $71.4(6.6)$ & $70.0(6.4)$ & $<0.001$ & $<0.001^{*}$ \\
\hline Sex & $221(78.9 \%)$ & $63(66.3 \%)$ & 0.014 & $0.017 \dagger$ & 340 (75.6\%) & $840(70.2 \%)$ & 0.033 & $0.004 \dagger$ \\
\hline Disease duration & $1.4(1.0)$ & $1.2(0.8)$ & 0.071 & $0.078 \ddagger$ & $1.3(1.0)$ & $1.3(0.9)$ & 0.76 & $0.60 \ddagger$ \\
\hline UPDRS 3 & $26.8(12.3)$ & $26.5(12.2)$ & 0.82 & $0.89 \S$ & $24.9(11.6)$ & $23.9(11.7)$ & 0.17 & $0.81 \S$ \\
\hline \multicolumn{9}{|l|}{ Motor subtype } \\
\hline TD & $108(42.0 \%)$ & $29(34.1 \%)$ & & & 230 (54.1\%) & $530(47.7 \%)$ & & \\
\hline PIGD & $116(45.1 \%)$ & $48(56.5 \%)$ & 0.11 & $0.16 \S$ & $144(33.9 \%)$ & $434(39.1 \%)$ & 0.031 & $0.009 \S$ \\
\hline Mixed & $33(12.8 \%)$ & $8(9.4 \%)$ & 0.82 & $0.58 \S$ & $51(12.0 \%)$ & $147(13.2 \%)$ & 0.22 & $0.39 \S$ \\
\hline \multicolumn{9}{|l|}{ MoCA } \\
\hline Total & $24.2(3.6)$ & $23.9(3.3)$ & 0.48 & $0.23 \S$ & $24.0(3.8)$ & $25.1(3.4)$ & $<0.001$ & $<0.001 \S$ \\
\hline Normal & $169(63.3 \%)$ & 47 (51.6\%) & 0.095 & $0.043 \S$ & $249(59.6 \%)$ & $831(73.8 \%)$ & $<0.001$ & $<0.001 \S$ \\
\hline $\mathrm{MCl}$ & $42(15.7 \%)$ & $22(24.2 \%)$ & & & $75(17.9 \%)$ & $149(13.2 \%)$ & & \\
\hline Dementia & $56(21.0 \%)$ & $22(24.2 \%)$ & & & $94(22.5 \%)$ & $146(13.0 \%)$ & & \\
\hline LEDD & $317(210)$ & $304(176)$ & 0.57 & $0.57 \S$ & $275(203)$ & $294(206)$ & 0.11 & $0.025 \S$ \\
\hline \multicolumn{9}{|l|}{ Smoking } \\
\hline Non-smoker & $109(44.7 \%)$ & $49(56.3 \%)$ & 0.12 & $0.20 \S$ & $215(52.1 \%)$ & $611(55.1 \%)$ & 0.31 & $0.41 \S$ \\
\hline Ex-smoker & $127(52.0 \%)$ & $33(37.9 \%)$ & & & $183(44.3 \%)$ & $457(41.2 \%)$ & & \\
\hline Current smoker & $8(3.3 \%)$ & $5(5.7 \%)$ & & & $15(3.6 \%)$ & $40(3.6 \%)$ & & \\
\hline
\end{tabular}

Data are mean (SD) or number (percentage) unless otherwise stated.

${ }^{*}$ Adjusted for disease duration and sex.

tAdjusted for age and disease duration.

$\ddagger$ Adjusted for age and sex.

$\S$ Adjusted for age, sex, disease duration and coffee use.

MCI, mild cognitive impairment; MoCA, Montreal cognitive assessment; PIGD, postural instability gait difficulty; TD, tremor dominant; UPDRS 3, Movement Disorder Society unified

Parkinson's disease rating scale part 3.

of major importance (ClinicalTrials.gov identifier NCT02787590).

One limitation of our study is that the NICE guideline threshold was amended (from QRISK2 $\geq 20 \%$ to QRISK2 $\geq 10 \%$ ) in July $2014,{ }^{11}$ which overlapped with our study recruitment. For this reason, we stratified our results by the prior $20 \%$ threshold (published in 2008) and the newer 10\% threshold. However, even considering the $20 \%$ threshold (37.2\% prescribed statins), there was a major difference in statin use compared with that in manifest CVD (75.3\% prescribed statins). A further limitation of the current study is that neuroimaging (eg, MRI or CT) was not used to assess vascular changes. While we have explored imaging in relation to individual risk factors elsewhere, our desired focus within this study was on the clinical assessment of vascular risk. We did not record statin dosage information, but this is in keeping with many studies which have explored statin use according to vascular risk indication, ${ }^{13} 1617$ and with many studies examining statin use in relation to the risk of PD. ${ }^{40} \mathrm{~A}$ final limitation of our study relates to diagnostic accuracy, which like other early PD studies was based on clinical diagnosis in a research framework using clinical diagnostic criteria. Although some cases may evolve to an alternative diagnosis, in most cases, vascular risk assessment and appropriately directed treatment remain relevant. For this reason, we included all patients in our assessment of vascular risk and treatment, and limited our analysis to patients without possible atypical features only when examining clinical correlates between vascular risk and disease, and PD phenotype.

In conclusion, a large proportion of individuals with recent-onset PD have increased cardiovascular risk, which is associated with greater motor and cognitive severity, and greater axial impairment. Statin therapy is underused in these PD cases, which contrasts with much high rates of statin use in PD cases with manifest CVD. Increasing the usage of statins in PD patients with increased vascular risk would reduce acute cardiovascular events, but might also reduce chronic vascular damage, and thereby slow the progression of motor and cognitive decline. Patients with PD have regular visits to healthcare providers, and so greater awareness and increased intervention in this group could have an immediate impact in a large population.

\section{Author affiliations}

${ }^{1}$ Department of Neurology, Institute of Neurological Sciences, Glasgow, UK ${ }^{2} S$ chool of Social and Community Medicine, University of Bristol, Bristol, UK ${ }^{3}$ Oxford Parkinson's Disease Centre, Nuffield Department of Clinical Neurosciences, University of Oxford, Oxford, UK

${ }^{4}$ Department of Neurology, Queen's Medical Centre, Nottingham, UK ${ }^{5}$ Clinical Neurosciences, John van Geest Centre for Brain Repair, Cambridge, UK ${ }^{6}$ Institute of Neuroscience, University of Newcastle, Newcastle upon Tyne, UK ${ }^{7}$ Sobell Department of Motor Neuroscience, UCL Institute of Neurology, London, UK ${ }^{8}$ Department of Clinical Neuroscience, UCL Institute of Neurology, London, UK ${ }^{9}$ Psychological Medicine and Clinical Neurosciences, MRC Centre for Neuropsychiatric Genetics and Genomics, Cardiff University, Cardiff, UK

${ }^{10}$ Department of Molecular Neuroscience, UCL Institute of Neurology, London, UK

${ }^{11}$ UK Clinical Consortium, 72 Clinical Centres across the UK

Acknowledgements The Tracking Parkinson's study is funded by Parkinson's UK, and supported by the National Institute for Health Research (NIHR) Dementias and Neurodegenerative Diseases Research Network (DeNDRoN), the NIHR Newcastle Biomedical Research Unit based at Newcastle upon Tyne Hospitals NHS Foundation Trust and Newcastle University, and the NIHR funded Biomedical Research Centre in Cambridge. The QRISK2 batch calculator used for this study was funded by Michael's Movers for Parkinson's. The Oxford Discovery study is funded by the Monument Trust Discovery Award from Parkinson's UK, and supported by the NIHR Oxford Biomedical Research Centre based at Oxford University Hospitals NHS Trust and University of Oxford and DeNDRoN. The Tracking Parkinson's study group was (principal investigators): DGG, NPB, L Sugathapala, DJB, A Graham, D Bathgate, R Bland, P Worth, G Mamutse, K Amar, R Walker, J Raw, C Carroll, CE Clarke, Z Hemsley, R Fackrell, H Roberts, S Guptha, U Nath, RAB, C Counsell, R Sheridan, M 
Silverdale, J Sharma, P Piccini, J Hindle, S Arianayagam, S Ellis, T Ward, G Lennox, M Carson, S Sveinbjornsdottir, B Boothman, D Paviour, A Misbahuddin, A Schrag, R Athey, P Sarda, M Steiger, Z Dhakam, N Kock, S Molloy, M O'Neill, J Stern, E Capps, P Critchley, TF, J George, O Bandmann, G Harper, T Andrews, W Woodward, A Whone, C Borland, M Wilson, Y Adenwala, P Tidswell, R Chaudhuri, A Watt, A Church, HRM, MTMH, S Kamath, B Adler, S Barber, E De Pablo-Fernandez, R Sophia; and (research nurses and healthcare professionals): V Agarwal, L Alderton, K Amor, A Andrew, S Arif, J Bennett, K Birchall, J Birt, K Blachford, J Brooke, A Brown, P Brown, C Brugaletta, N Bryden, M Burrows, S Butler, S Cable, R Callaghan, L Canovas, G Carey, L Cattarall, K Clipsham, W Colwell, Z Cowen, C Cox, S Craw, A Creaser-Smith, Y Croucher, S Daniel, A De Pietro, D Dellafera, S Dodds, A Donaldson, D Donaldson, A Dougherty, C Downes, S Dube, W Dwornik, C Edwards, E Ekins, R Fernandes, C Foale, H Forbes, S Ford, J Frost, T Fuller, L Gallagher, R Gentle, L Gethin, J Gilford, C Gray, E Gunter, S Hall, C Hamilton, M Hare, A Henderson, V Hetherington, R Higgins, A Higham, L Hill, K Hodgson, $R$ Humphries, S Hurlstone, A Hursey, R Inniss, R James, E Johnson, R Joyce, Z Kefalopoulou, M Kelly, M Korley, A Lehn, S Levy, K Lithgo, C Long, A Lyle, H Lynn, L Mackinnon, C Makahamadze, T Mahan, N Marks, S Marrinan, M Marshall, P Martin-Forbes, I Massey, C McBrearty, J McEntee, A McNichol, D Mills, S Morgan, D Mullan, T Murphy, J Newman, H O'Connell, A O'Donnell, D O'Donnell, C O'Reilly, O Olanrewaju, E Oughton, C Owen, S Painter, S Palfreeman, P Paterson, L Perkins, A Pilcher, K Powell, C Price, P Rachman, L Renton, J Rickett, A Rizos, T Roberts, M Roche, R Roopun, A Roussakis, R Rowland, G Saunders, C Sequeira, S Shields, D Simmons, C Snape, J Stickley, L Strong, C Sunderland, S Sutherland, N Temple, E Thomson, M Trimmer, J Tuazon, E Tyrrell, E Visentin, C Vandor, N Vernon, N Verstraelen, M Visick, H Walsh, S Walsh, K Ward, A Watson, A Watt, E Whelan, J Williams, M Williams, SWilliams, B Wilson, K Witherington, R Woodcock, L Wyatt.

Contributors DMAS contributes to data analysis, manuscript writing and editing; MAL contributes to data analysis, manuscript editing; KAG and DGG contribute to the study design, data collection and analysis, manuscript writing and editing; NM contributes to data collection and manuscript editing; $F B, C R, J K, N P B, R A B, D J B$, TF, HRM and MTMH contribute to the study design, data collection and manuscript editing; YB-S contributes to the study design, manuscript editing; NW and NWW contribute to the study design.

Funding National Institute for Health Research (NIHR) Dementias and Neurodegenerative Diseases Research Network (DeNDRoN); NIHR Biomedical Research Centre in Cambridge; NIHR Oxford Biomedical Research Centre; NIHR Newcastle Biomedical Research Unit; Parkinson's UK (grant number 10.13039/ 501100000304); Michael's Movers for Parkinson's (Registered Charity No: SC042915). Parkinson's UK grant numbers for the Tracking Parkinson's and Oxford discovery cohort are J-1101 and J-1403 respectively.

Competing interests NPB has received payment for advisory board attendance from UCB, Teva Lundbeck, Britannia, GSK, Boehringer and honoraria from UCB Pharma, GE Healthcare, Lily Pharma, Medtronic. He has received research grant support from GE Healthcare, Wellcome Trust, MRC and Parkinson's UK and royalties from Wiley. RAB has received grants from Parkinson's UK, NIHR, Cure Parkinson's Trust, Evelyn Trust, Rosetrees Trust, MRC and EU along with payment for advisory board attendance from Oxford Biomedica and LCT, and honoraria from Wiley and Springer. DJB has received grants from NIHR, Wellcome Trust, GlaxoSmithKline, Parkinson's UK and Michael J Fox Foundation. He has acted as consultant for GSK. TF has received payment for advisory board meetings for Abbvie and Oxford Biomedica, and honoraria for presentations at meetings sponsored by Medtronic, St Jude Medical, Britannia and Teva pharmaceuticals. HRM has received grants from Medical Research Council UK, Wellcome Trust, Parkinson's UK, Ipsen Fund, Motor Neurone Disease Association, Welsh Assembly Government, PSP Association, CBD Solutions and Drake Foundation, and payment for advisory board attendance and lectures from Acorda, Teva, AbbVie, Medtronic, Boehringer Ingelheim, UCB and GSK. MTMH has received grants from Parkinson's UK, Michael J Fox Foundation, GE Healthcare, NIHR and Cure Parkinson's Trust. DGG has received grants from Parkinson's UK, Michael's Movers, the Paul Hamlyn Foundation, payment for advisory board attendance from AbbVie and honoraria from UCB Pharma, GE Healthcare and Acorda.

Ethics approval Multicentre ethics committee and local research and development approvals for each site.

Provenance and peer review Not commissioned; externally peer reviewed.

Open Access This is an Open Access article distributed in accordance with the terms of the Creative Commons Attribution (CC BY 4.0) license, which permits others to distribute, remix, adapt and build upon this work, for commercial use, provided the original work is properly cited. See: http://creativecommons.org/licenses/ by/4.0/

\section{REFERENCES}

1 Hajjar I, Quach L, Yang F, et al. Hypertension, white matter hyperintensities, and concurrent impairments in mobility, cognition, and mood: the Cardiovascular Health Study. Circulation 2011;123:858-65.
2 Verdelho A, Madureira S, Ferro JM, et al. Differential impact of cerebral white matter changes, diabetes, hypertension and stroke on cognitive performance among non-disabled elderly. The LADIS study. J Neurol Neurosurg Psychiatry 2007:78:1325-30.

3 Veselý B, Antonini A, Rektor I. The contribution of white matter lesions to Parkinson's disease motor and gait symptoms: a critical review of the literature. J Neural Transm (Vienna) 2016;123:241-50.

4 Jones JD, Jacobson C, Murphy M, et al. Influence of hypertension on neurocognitive domains in nondemented Parkinson's disease patients. Parkinsons Dis 2014; 2014:507529.

5 Bohnen NI, Kotagal V, Müller ML, et al. Diabetes mellitus is independently associated with more severe cognitive impairment in Parkinson disease. Parkinsonism Relat Disord 2014:20:1394-8.

6 Kotagal V, Albin RL, Müller ML, et al. Diabetes is associated with postural instability and gait difficulty in Parkinson disease. Parkinsonism Relat Disord 2013; 19:522-6

7 Papapetropoulos S, Ellul J, Argyriou AA, et al. The effect of vascular disease on late onset Parkinson's disease. Eur J Neurol 2004;11:231-5.

8 Kandiah N, Zainal NH, Narasimhalu K, et al. Hippocampal volume and white matter disease in the prediction of dementia in Parkinson's disease. Parkinsonism Relat Disord 2014;20:1203-8.

9 Gallardo MJ, Cabello JP, Pastor C, et al. Patients with advanced Parkinson's disease with and without freezing of gait: a comparative analysis of vascular lesions using brain MRI. Neurologia 2014;29:218-23.

10 Jellinger KA. Prevalence of cerebrovascular lesions in Parkinson's disease. A postmortem study. Acta Neuropathol 2003;105:415-19.

11 NICE. Lipid modification: cardiovascular risk assessment and the modification of blood lipids for the primary and secondary prevention of cardiovascular disease. 2014. http://www.nice.org.uk/guidance/cg181 (cited June 2015).

12 Wang Q, Yan J, Chen $X$, et al. Statins: multiple neuroprotective mechanisms in neurodegenerative diseases. Exp Neurol 2011;230:27-34.

13 Sheppard JP, Fletcher K, McManus RJ, et al. Missed opportunities in prevention of cardiovascular disease in primary care: a cross sectional study. $\mathrm{Br} / \mathrm{Gen}$ Pract 2014;64:e38-46.

14 Zhang H, Plutzky J, Skentzos S, et al. Discontinuation of statins in routine care settings: a cohort study. Ann Intern Med 2013;158:526-34.

15 Monaldi B, Bologna G, Costa GG, et al. Adherence to statin treatment following a myocardial infarction: an Italian population-based survey. Clinicoecon Outcomes Res 2015;7:273-80.

16 Kotseva K, Wood D, De Backer G, et al on behalf of EUROASPIRE Study Group. EUROASPIRE III management of cardiovascular risk factors in asymptomatic high-risk patients in general practice: cross-sectional survey in 12 European countries. Eur J Cardiovasc Prev Rehabil 2010;17:530-40.

17 Murphy C, Bennett K, Fahey T, et al. Statin use in adults at high risk of cardiovascular disease mortality: cross-sectional analysis of baseline data from The Irish Longitudinal Study on Ageing (TILDA). BMJ Open 2015;5:e008017.

18 Jaussi $A$, Noll G, Meier B, et al. Current cardiovascular risk management patterns with special focus on lipid lowering in daily practice in Switzerland. Eur J Cardiovasc Prev Rehabil 2010;17:363-72.

19 Malek N, Swallow DM, Grosset KA, et al. Tracking Parkinson's: study design and baseline patient data. J Parkinsons Dis 2015;5:947-59.

20 Szewczyk-Krolikowski K, Tomlinson P, Nithi K, et al. The influence of age and gender on motor and non-motor features of early Parkinson's disease: initial findings from the Oxford Parkinson Disease Center (OPDC) discovery cohort. Parkinsonism Relat Disord 2014;20:99-105.

21 NICE. Cardiovascular disease: risk assessment and reduction, including lipid modification. 2014. http://www.nice.org.uk/guidance/cg181 (cited May 2016).

22 Hippisley-Cox J, Coupland C, Vinogradova Y, et al. Derivation and validation of QRISK, a new cardiovascular disease risk score for the United Kingdom: prospective open cohort study. BMJ 2007;335:136.

23 Stebbins GT, Goetz CG, Burn DJ, et al. How to identify tremor dominant and postural instability/gait difficulty groups with the movement disorder society unified Parkinson's disease rating scale: comparison with the unified Parkinson's disease rating scale. Mov Disord 2013:28:668-70.

24 Dalrymple-Alford JC, MacAskill MR, Nakas CT, et al. The MoCA. Well suited screen for cognitive impairment in Parkinson Disease. Neurology 2010;75:1717-25.

25 Tomlinson $\mathrm{CL}$, Stowe R, Patel S, et al. Systematic review of levodopa dose equivalency reporting in Parkinson's disease. Mov Disord 2010;25:2649-53.

26 Rektor I, Goldemund D, Sheardová K, et al. Vascular pathology in patients with idiopathic Parkinson's disease. Parkinsonism Relat Disord 2009;15:24-9.

$27 \mathrm{Li} \mathrm{HJ}, \mathrm{Yu}$ Y, Chen Y, et al. Vascular risk factors aggravate the progression of Parkinson's disease: a five-year follow-up study in Chinese patients. Int I Clin Exp Med 2015;8:9897-903.

28 de Bruijn RF, Ikram MA. Cardiovascular risk factors and future risk of Alzheimer's disease. BMC Med 2014;12:130.

29 Tettey P, Simpson SJr, Taylor BV, et al. Vascular comorbidities in the onset and progression of multiple sclerosis. J Neurol Sci 2014;347:23-33. 
30 Kotagal V, Albin RL, Müller ML, et al. Advanced age, cardiovascular risk burden, and timed up and go test performance in Parkinson disease. J Gerontol A Biol Sci Med Sci 2014;69:1569-75.

31 Kotagal V, Albin RL, Müller ML, et al. Modifiable cardiovascular risk factors and axial motor impairments in Parkinson disease. Neurology 2014;82:1514-20.

32 Cereda E, Barichella M, Cassani E, et al. Clinical features of Parkinson disease when onset of diabetes came first: a case-control study. Neurology 2012;78:1507-11.

33 Banach M, Rizzi M, Toth PP, et al. Statin intolerance - an attempt at a unified definition. Position paper from an International Lipid Expert Panel. Arch Med Sci 2015;11:1-23.

34 Xiong $Y$, Wong $A$, Cavalieri $M$, et al. Prestroke statins, progression of white matter hyperintensities, and cognitive decline in stroke patients with confluent white matter hyperintensities. Neurotherapeutics 2014;11:606-11.
35 Valenti R, Pantoni L, Markus HS. Treatment of vascular risk factors in patients with a diagnosis of Alzheimer's disease: a systematic review. BMC Med 2014;12:160.

36 Evans JR, Mason SL, Williams-Gray CH, et al. The natural history of treated Parkinson's disease in an incident, community based cohort. J Neurol Neurosurg Psychiatr 2011;82:1112-18.

37 Saher G, Simons M. Cholesterol and myelin biogenesis. Subcell Biochem 2010;51:489-508.

38 Langsjoen PH, Langsjoen AM. The clinical use of HMG CoA-reductase inhibitors and the associated depletion of coenzyme Q10. A review of animal and human publications. Biofactors 2003;18:101-11.

39 Ott BR, Daiello LA, Dahabreh IJ, et al. Do statins impair cognition? A systematic review and meta-analysis of randomized controlled trials. J Gen Intern Med 2015;30:348-58

40 Bai $S$, Song $Y$, Huang $X$, et al. Statin use and the risk of Parkinson's disease: an updated meta-analysis. PLoS One 2016;11:e0152564. 